\title{
PHOTOPRODUCTION OF NEUTRAL RHO MESONS FROM COMPLEX NUCLEI
}

H. Alvensleben, U. Becker, William K. Bertram, M. Chen, K. J. Cohen,

T. M. Knasel, R. Marshall, D. J. Quinn, M. Rohde, G. H. Sanders, H. Schubel, and Samuel C. C. Ting

Deutsches Elektronen Synchrotron, Hamburg, Germany, and Department of Physics and Laboratory for Nuclear Science, Massachusetts Institute of Technology, Cambridge, Massachusetts 02139 (Received 30 October 1969)

\begin{abstract}
We present results of measurements on photoproduction of $\rho$ mesons. Analysis of $10^{6}$ measured $\rho$ events in a four-dimensional data matrix $d \sigma\left(A, m, p, t_{\perp}\right) / d \Omega d m$ with dimensions $14 \times 20 \times 10 \times 20$ yields precise information on nuclear density distributions for $\rho$ production. We obtain for the Woods-Saxon radii $R(A)=(1.12 \pm 0.02) A^{1 / 3}$ and, using the vector dominance model, $\sigma_{\rho N}=26.7 \pm 2.0 \mathrm{mb}$ and $\gamma_{\rho}{ }^{2} / 4 \pi=0.57 \pm 0.10$.
\end{abstract}

We present results on the photoproduction of neutral $\rho$ mesons, ${ }^{1}$

$$
\gamma^{+} A \rightarrow A+\rho
$$

from 14 elements: hydrogen $(A=1)$, beryllium (9), carbon (12), aluminum (27), titanium (47.9), copper (63.5), silver (107.9), cadmium (112.4), indium (114.7), tantalum (181), tungsten (183.9), gold (197), lead (207.2), and uranium (238.1). The measurements covered 20 intervals in the di-pion mass $(m)$ region from 400 to $1000 \mathrm{MeV} /$ $c^{2}, 10$ intervals in the $\rho$ momentum $(p)$ from 3.5 to $7 \mathrm{GeV} / c$, and 20 intervals in the transverse momentum transfer to the nucleus $\left(t_{\perp}\right)$ from 0.0 to $-0.04(\mathrm{GeV} / c)^{2}$. These measurements form a four-dimensional data matrix $\left(A, m, p, t_{\perp}\right)$, $14 \times 20 \times 10 \times 20$, containing approximately one million measured $\pi^{+} \pi^{-}$events. The high statistics of this experiment (between $10^{2}$ and $10^{3}$ times more events than previous work) together with the large variety of elements used (twice that of previous experiments) $)^{2,3}$ enables us to make an accurate study of the following:

(1) The nuclear density distributions. For a given $A$, the $t$ dependence yields information (to $\pm 2 \%$ accuracy) on the size of the nuclear radius $R(A)$ seen by the $\rho$ meson.

(2) The absolute and relative forward $\rho$-production cross sections $d \sigma\left(A, m, p, t_{\perp}\right) / d \Omega d m$. For fixed $A, p$, and $t_{\perp}$, measurement of the $\pi \pi$ spectra as a function of mass alone provides a unique determination of the $\rho$ line shape and the background and hence an accurate determination of the cross section.

(3) The $\rho$-nucleon cross section $\sigma_{\rho N}$ and the $\gamma-\rho$ coupling constant $\gamma_{\rho}{ }^{2} / 4 \pi$. Measurement of the nuclear density distributions and the production cross sections from the 14 elements determines the rate of reabsorption of $\rho$ by nuclear matter and the effective forward production cross sec- tion per nucleon $\left|f_{0}\right|^{2}$. This yields $\sigma_{\rho N}$ and $\gamma_{\rho}{ }^{2} /$ $4 \pi=(\alpha / 64 \pi)\left(\sigma_{\rho N}{ }^{2} /\left|f_{0}\right|^{2}\right)$ in a self-consistent manner. Since the original work of Lanzerotti et al., ${ }^{1}$ several experiments have been done ${ }^{2,3}$ to investigate reaction (1) in order to determine $\sigma_{\rho N}$ and $\gamma_{\rho}{ }^{2} / 4 \pi$. They have obtained different results and some of the results are different from the predictions of the vector dominance $\operatorname{model}^{4}\left(\gamma_{\rho}^{2} / 4 \pi\right.$ $\left.\simeq 0.5 ; \quad \sigma_{\rho N} \simeq \sigma_{\pi N}\right)$.

The present experiment was carried out at the 7.5-GeV DESY electron synchrotron. A bremsstrahlung beam interacted in the target and the photoproduced pairs were detected by a largeaperture magnetic spectrometer described previously. ${ }^{5}$ Vacuum pipes and helium filled bags were placed inside the spectrometer to reduce miltiple scattering and nuclear absorption of pions. The 22500 hodoscope combinations defined an event to an accuracy of $\Delta m= \pm 15 \mathrm{MeV} /$ $c^{2}, \Delta p= \pm 150 \mathrm{MeV} / c$, and $\Delta t_{\perp}= \pm 0.001(\mathrm{GeV} / c)^{2}$.

During the experiment many precautions were taken to ensure that the spectrometer behaved as designed and that all systematic effects were understood. We list the following 10 examples:

(1) To ensure that the data are not sensitive to second order effects in the target, such as photon beam attenuation and pion absorption, we measured the rate of reaction (1) as a function of target thickness from 0 to $5 \mathrm{~g} / \mathrm{cm}^{2}$ of carbon. Within an accuracy of $1 \%$, the corrected counting rate increased linearly with target thickness.

(2) Accidental coincidences were monitored by a series of duplicate logic circuits of different resolving times and were kept below $2 \%$ by controlling the beam intensity.

(3) The dead time of the electronics was monitored by continuously recording the single rates in the counters. The beam intensity was adjusted such that the dead time was less than $2 \%$.

(4) Nuclear absorption of pions by material in the spectrometer was investigated by introducing 
additional material and by varying the gas pressure in the Cherenkov counters. The measured loss of $\pi$ pairs agreed with calculations based on published data. ${ }^{6}$

(5) To avoid any possible effects from spectrometer asymmetry, half the data were taken at each polarity of the spectrometer The rates from the two spectrometer polarities were identical.

(6) All counter voltages were kept constant to $\pm 5 \mathrm{~V}$ and all magnetic fields were kept stable to 3 parts in $10^{4}$.

(7) To ensure that low-mass $\pi$ pairs from high$Z$ elements are not contaminated by $e^{+} e^{-}$pairs, Cherenkov counters were used to count and to reject the $e^{+} e^{-}$pairs. They indicated that the maximum contamination was less than one part in $10^{4}$.

(8) To ensure the reproducibility of the data, normalization runs were made every few hours. Over the entire running period the system was reproducible to $\pm 1 \%$.

(9) To keep errors from inelastic contributions to reaction (1) small, all the data were taken with $p$ close to the peak photon energy $k_{\max }$ $\left(k_{\text {max }} / p \simeq 1.2\right)$.

(10) The purity of the targets was chosen to be better than $99.9 \%$. The thickness of the targets was chosen such that the corrections for beam attenuation and for pion absorption were similar for each element and that the target-out rates were small.

The data were corrected for small systematic effects such as beam attenuation, target out, nuclear absorption, dead time, accidentals, etc. All of these corrections were checked by measurement in the same spectrometer to be accurate to $1 \%$. The spectrometer acceptance was calculated by a Monte Carlo method using fourth-

$$
\frac{d \sigma}{d \Omega d m}\left(A, m, p, t_{\perp}\right)=\frac{1}{\pi} p^{2} 2 m R_{n}(m)\left(f_{c}+f_{\mathrm{inc}}\right)+f_{\mathrm{B} \mathrm{G}}\left(A, m, p, t_{\perp}\right) \text {. }
$$

The first term represents the main contribution from $\rho$ photoproduction and the second term the contribution due to a nonresonant background;

$$
\begin{aligned}
& f_{c}=f_{c}\left(A, t_{\perp}, t_{\|}, \sigma, \beta\right)=\left(\sigma^{\prime} / \sigma\right)^{2} \mid 2 \pi f_{0} \int_{0}^{\infty} d b \int_{-\infty}^{\infty} d z b J_{0}\left(b \sqrt{ }\left|t_{\perp}\right|\right) \exp \left(i z \sqrt{ }\left|t_{\|}\right|\right) \rho(z, b) \\
& \times\left.\exp \left[-\frac{1}{2} \sigma^{\prime}(1-i \beta) \int_{z}^{\infty} \rho\left(z^{\prime}, b\right) d z^{\prime}\right]\right|^{2}, \\
& \sigma^{\prime}=\sigma(1-\xi \eta \sigma), \quad \xi=1 / 16 \pi a \int \exp \left(-b^{2} / 4 a\right) g(b, z) d^{2} b d z, \\
& \eta=\eta(\sigma)=\int \exp \left[-\frac{1}{2} \sigma T(b)\right] Q(b) d^{2} b\left\{\int \exp \left[-\frac{1}{2} \sigma T(b)\right] T(b) d^{2} b\right\}^{-1}, \\
& Q(b)=\int_{-\infty}^{\infty} \rho^{2}(b, z) d z, \quad T(b)=\int_{-\infty}^{\infty} \rho(b, z) d z, \quad \sigma=\sigma_{\rho N}, \quad a=8(\mathrm{GeV} / c)^{-2} .
\end{aligned}
$$




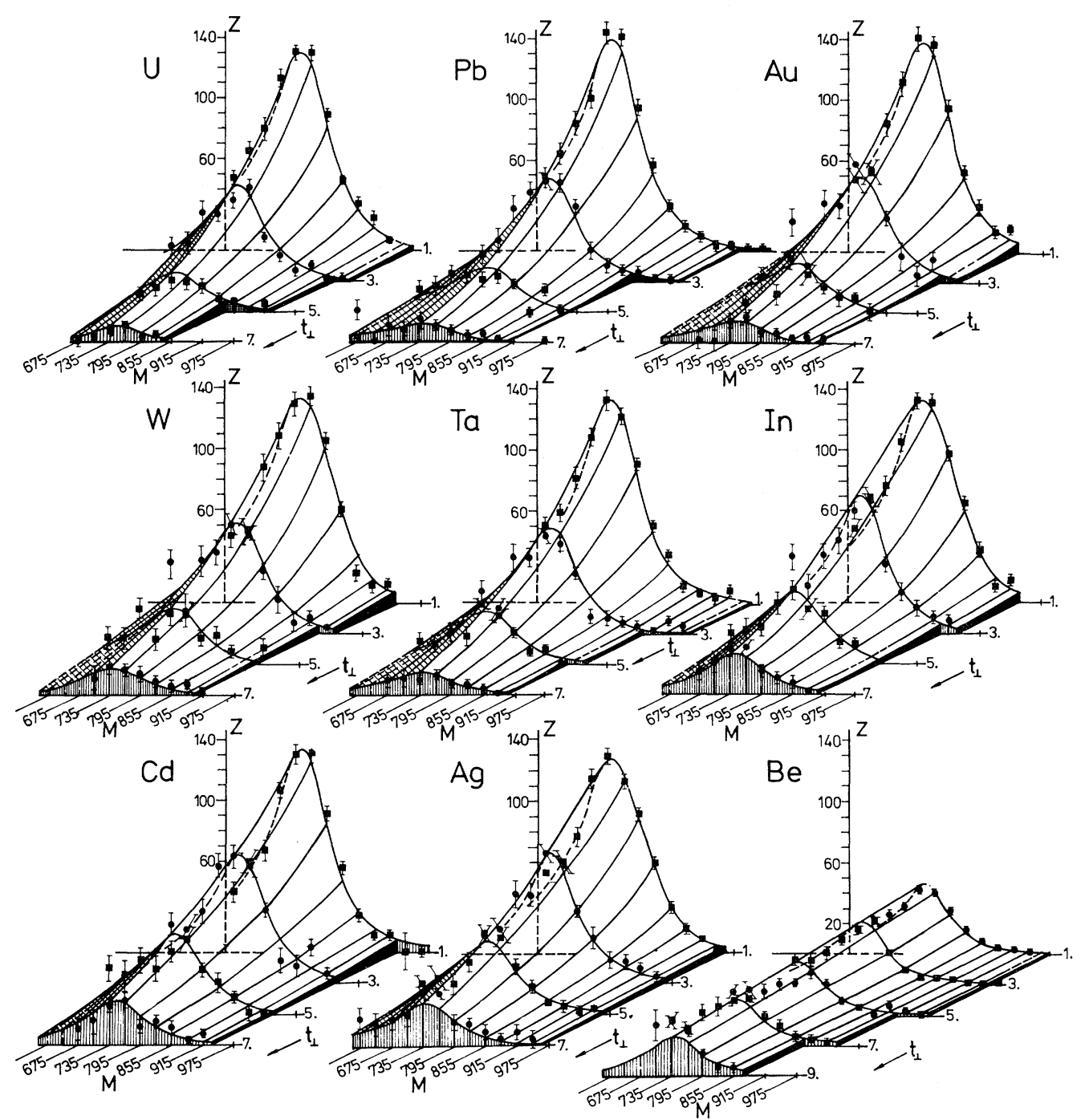

FIG. 1. The cross section $Z=d \sigma / d \Omega d m\left[\mu \mathrm{b} / \mathrm{sr}\left(\mathrm{MeV} / c^{2}\right)\right.$ nucleon] as a function of $m\left(\mathrm{MeV} / c^{2}\right)$ and $t_{\perp}$ in units of $-0.001 \mathrm{GeV}^{2} / c^{2}$ for $p=6.2 \pm 0.2 \mathrm{GeV} / c$. The curves are the best fits to Eq. (2) with $R_{1}(m)$. The background is not drawn. This figure shows about $2 \%$ of the data.

$f_{c}$ is the coherent production cross section, ${ }^{9}$ where the $\rho$ meson, produced with an effective forward production cross section $\left|f_{0}\right|^{2}$ on a single nucleon, is attenuated by $\exp \left[-\frac{1}{2} \sigma^{\prime}(1-i \beta)\right.$ $\left.\times \int_{z}^{\infty} \rho d z\right]$ in nuclear matter as it leaves the nucleus. The factor $\rho(b, z) J_{0}\left(b \checkmark\left|t_{1}\right|\right)$ comes from the nuclear shape; $\rho=\rho_{0}\{1+\exp [(r-R) / s]\}^{-1}$ is the Woods-Saxon density, where $R$ is the nuclear radius and $s=0.545 \mathrm{~F} . \operatorname{Exp}\left(i z \sqrt{ }\left|t_{\|}\right|\right)$comes from the difference in initial and final mass, and $\sigma^{\prime}$ is the effective $\rho$-nucleon total cross section where we have taken into account second order correlation effects between nucleons inside the nucleus. $\xi$ is the correlation length and $g(b, z)$ the correlation wave function. ${ }^{10} \beta$ is the ratio of real part to imaginary part of the scattering amplitude on a nucleon. The value $\beta=-0.2$ was taken from the analysis of $\gamma p$ total cross section measurements at $6.0 \mathrm{GeV} .{ }^{11} f_{\mathrm{inc}}$ is the incoherent production cross section where the recoil nucleus is left in an excited or fragmented state. ${ }^{12}$ The incoherent contribution is largest for low $A(\simeq 10 \%)$ and becomes negligible for $A>100$. The background function $f_{\mathrm{BG}}\left(A, m, p, t_{\perp}\right)$ is a general polynomial in $\left(A, m, p, t_{\perp}\right)$ space.

$2 m R_{n}(m)$ are relativistic Breit-Wigner mass distributions. To keep the results general, we tried various commonly used forms for the BreitWigner and we list the following five examples:

$$
r(m, \Gamma(m))=r(m)=\frac{1}{\pi} \frac{m_{\rho} \Gamma(m)}{\left(m_{\rho}^{2}-m^{2}\right)^{2}+m_{\rho}{ }^{2} \Gamma^{2}(m)},
$$




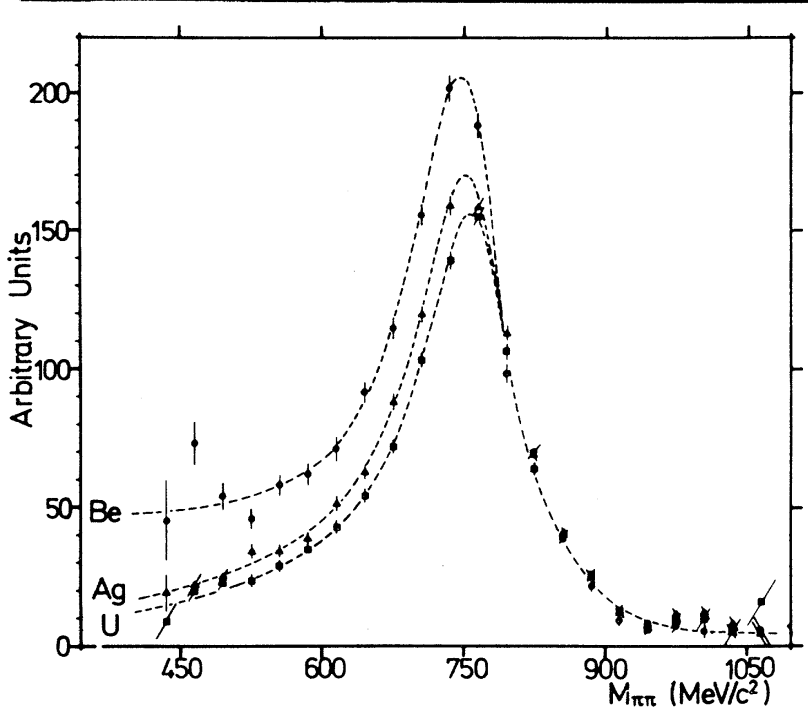

FIG. 2. Mass spectra after removal of production mechanism. (Note that in the absence of background, all spectra would be identical.)

$$
\begin{aligned}
& \Gamma(m)=\frac{m_{\rho}}{m}\left[\frac{(m / 2)^{2}-m_{\pi}^{2}}{\left(m_{\rho} / 2\right)^{2}-m_{\pi}^{2}}\right]^{3 / 2} \Gamma_{0}, \\
& I(m)=\frac{D}{2 m} \frac{m^{2}-m_{\rho}^{2}}{\left(m_{\rho}^{2}-m^{2}\right)^{2}+m_{\rho}^{2} \Gamma^{2}(m)}, \\
& r_{0}(m)=r\left(m, \Gamma_{0}\right), \\
& \boldsymbol{R}_{1}(m)=r(m)\left(m_{\rho} / m\right)^{4}, \quad \boldsymbol{R}_{2}(m)=r(m)+I(m), \\
& \boldsymbol{R}_{3}(m)=r(m)\left(m_{\rho} / m\right)^{4}+I(m), \quad \boldsymbol{R}_{4}(m)=r_{0}(m), \\
& \boldsymbol{R}_{5}(m)=r(m),
\end{aligned}
$$

$R_{5}(m)$ is the relativistic $p$ wave resonance for mula proposed by Jackson, ${ }^{13} R_{4}(m)$ is $R_{5}(m)$ with a constant width. $R_{1}(m)$ includes a modification by a phenomenological "Ross-Stodolsky" ter m ${ }^{14}$ $\left(m_{\rho} / m\right)^{4}$. The factor $\left(m_{\rho} / m\right)^{4}$ also appears in a model by Kramer and Uretsky, ${ }^{14}$ and has been used widely to account for the shift and shape distortion of the photoproduced $\rho$ spectrum. $R_{2}(m)$ represents another commonly accepted procedure to account for the mass shift and shape distortion by a "Söding" interference term $I(m),{ }^{14}$ whose magnitude $D$ is determined by the fit. $R_{3}(m)$ assumes both mechanisms to be present.

Comparison of the 56000 data bins in the fourdimensional measured cross-section matrix with the theoretical function (2) enables us to determine directly the parameters $m_{\rho}, \Gamma_{0}, \sigma_{\rho N}, R(A)$, $\left|f_{0}\right|^{2}, d \sigma_{n}(A) /\left.d t\right|_{\theta=0^{\circ}}$.

The fitting was done with the CERN program MINUIT ${ }^{15}$ To reduce the contributions from the incoherent term $f_{\mathrm{inc}}$ and the background, we selected data in the region $\left|t_{\perp}\right|<\left|t_{c}\right|$ with $t_{c}$ $=-0.01(\mathrm{GeV} / c)^{2}, 5.0<p<7.0 \mathrm{GeV} / c$, and $m>m_{c}$ with $m_{c}=600 \mathrm{MeV} / c^{2}$. Thus we restricted the data to the region where most of the $\pi$ pairs come from coherent $\rho$ production. The determination of the various parameters can be visualized simply in the following way:

(1) To determine the background function $f_{\mathrm{BG}}(A$, $\left.m, p, t_{\perp}\right):$ Fits were made with and without a background term of the form

$$
\begin{aligned}
& f_{\mathrm{BG}}\left(A, m, p, t_{\perp}\right)=\left[\sum_{i=0}^{l} a_{i}(A) m^{I}\right] {\left[\sum_{j=0}^{m} b_{j}(A) p^{j}\right] } \\
& \times\left[\sum_{k=0}^{n} c_{k}(A) t_{\perp}{ }^{k}\right] .
\end{aligned}
$$

The goodness of fit improved considerably for $l=2, m=n=0$ (see Fig 1 ). Thereafter no improvement was noticed by increasing $l, m$, or $n$. The results of the fits were insensitive to changes in $m_{c}\left( \pm 100 \mathrm{MeV} / c^{2}\right)$ or $\left|t_{c}\right|<0.01(\mathrm{GeV} / c)^{2}$. The percentage background is considerable for low $A$, but decreases with increasing $A$ and $m$, being small on uranium (see Fig. 2).

(2) To determine $R(A)$, the nuclear density parameter for fixed $A$ : The $t$ dependence of the data measures the diffraction pattern of $\pi$ pairs off the nucleus. Comparing the diffraction pattern with Eq. (2) determines the radius seen by the $\rho$ meson. Table I shows the results of the measurements of the Woods-Saxon radii. They are insensitive to $s, \beta, m_{0}$, and $\Gamma_{0}$ and independent of the normalization and the vector dominance model. Being determined from neutral rho mesons, they are free from complications of Coulomb interference. The data yield $R(A)=(1.12+0.02) A^{1 / 3}$ $\mathrm{F}$ which is the most accurate determination of strong-interaction nuclear radii to date. ${ }^{16}$ Our radii are to be compared with those determined from electron scattering $\left[R(A) \simeq 1.08 A^{1 / 3} \mathrm{~F}\right] .^{17}$ A full discussion on the radius determination is to be presented in a later paper.

(3) To determine $m_{\rho}$ and $\Gamma_{0}$, the mass and width of $\rho$ : For fixed $A, p, t_{\perp}$, a comparison of Eq. (2) with the mass dependence of $d \sigma / d \Omega d m$ measures directly the mass and width of the $\rho$. Independent of the form of the mass distribution used, the width $\Gamma_{0}=140 \pm 5 \mathrm{MeV} / c^{2}$. Table I shows the fitted results for $m_{\rho}$. The mass $m_{\rho}$ varies from 740 to $775 \mathrm{MeV} / c^{2}$, depending on the exact forms of $R_{n}(m)$ used. The best fit values from $R_{1,2,3}(m)$ yield $w_{\rho}=765 \pm 10 \mathrm{MeV}$.

(4) To determine the coherent cross section $d \sigma_{n}(A) /\left.d t\right|_{\theta=0 \circ}$ the data matrix was compared 
Table I. Summary of measured radii $R(A)$, typical cross sections $\Sigma_{n}=d \sigma / d t\left(\theta=0^{\circ}, t \|=-0.002, p=6.54\right)$ $\left[\mu \mathrm{b} /(\mathrm{GeV} / c)^{2}\right.$ nucleon], $\chi^{2}(A) / D F(A),\left|f_{0}\right|^{2}, \sigma_{\rho N}$, and $\gamma_{\rho}^{2} / 4 \pi$. The cross sections do not include an uncertainty of $\pm 5 \%$ due to the normalization of the mass distribution $R_{n}(m)$. The errors include uncertainties in $R(A), m_{\rho}, \Gamma_{0}$, and $f_{\mathrm{BG}}$.

\begin{tabular}{|c|c|c|c|c|c|c|}
\hline A & $R(A) f$ & $\Sigma_{1}$ & $\Sigma_{2}$ & $\Sigma_{3}$ & $\Sigma_{4}$ & $\Sigma_{5}$ \\
\hline Beryllium & $2.35 \pm 0.26$ & $627 \pm 31$ & $652 \pm 50$ & $678 \pm 20$ & $628 \pm 20$ & $581 \pm 59$ \\
\hline Carbon & $2.50 \pm 0.23$ & $772 \pm 52$ & $800 \pm 50$ & $822 \pm 40$ & $767 \pm 31$ & $692 \pm 101$ \\
\hline Aluminium & $3.37 \pm 0.16$ & $1322 \pm 63$ & $1279 \pm 51$ & $1348 \pm 36$ & $1319 \pm 46$ & $1287 \pm 87$ \\
\hline Titanium & $3.94 \pm 0.10$ & $1796 \pm 78$ & $1706 \pm 66$ & $1749 \pm 44$ & $1760 \pm 102$ & $1622 \pm 84$ \\
\hline Copper & $4.55 \pm 0.11$ & $2099 \pm 115$ & $2102 \pm 68$ & $2179 \pm 60$ & $2203 \pm 77$ & $2137 \pm 196$ \\
\hline Silver & $5.35 \pm 0.09$ & $2591 \pm 79$ & $2585 \pm 73$ & $2631 \pm 61$ & $2725 \pm 139$ & $2594 \pm 177$ \\
\hline Cadmium & $5.40 \pm 0.14$ & $2636 \pm 93$ & $2583 \pm 74$ & $2662 \pm 60$ & $2801 \pm 90$ & $2584 \pm 113$ \\
\hline Indium & $5.56 \pm 0.25$ & $2696 \pm 90$ & $2634 \pm 74$ & $2713 \pm 50$ & $2801 \pm 157$ & $2651 \pm 115$ \\
\hline Tantalum & $6.50 \pm 0.15$ & $2938 \pm 154$ & $2900 \pm 131$ & $2980 \pm 124$ & $3049 \pm 180$ & $2903 \pm 199$ \\
\hline Tungsten & $6.30 \pm 0.12$ & $2925 \pm 140$ & $2877 \pm 75$ & $2959 \pm 76$ & $3045 \pm 112$ & $3035 \pm 229$ \\
\hline Gold & $6.45 \pm 0.27$ & $2948 \pm 128$ & $2966 \pm 147$ & $3017 \pm 69$ & $3118 \pm 103$ & $3025 \pm 111$ \\
\hline Lead & $6.82 \pm 0.20$ & $3112 \pm 93$ & $3167 \pm 76$ & $3234 \pm 59$ & $3368 \pm 174$ & $3165 \pm 161$ \\
\hline Uranium & $6.90 \pm 0.14$ & $3070 \pm 93$ & $3035 \pm 58$ & $3144 \pm 45$ & $3235 \pm 91$ & $3144 \pm 114$ \\
\hline$\chi^{2}(A) / D F(A)$ & - & $\sim 1.2$ & $\sim 1.2$ & $\sim 1.2$ & $\sim 1.5$ & $\sim 2.4$ \\
\hline$m_{p}\left(M_{e} V / c^{2}\right)$ & - & $765 \pm 10$ & $773 \pm 10$ & $765 \pm 10$ & $743 \pm 10$ & $742 \pm 10$ \\
\hline$\left|f_{0}\right|^{2} \mu b /(G e V / c)^{2}$ & - & $118 \pm 6$ & $120 \pm 7.4$ & $125.3 \pm 9$ & $112 \pm 6$ & $103 \pm 10$ \\
\hline$\sigma_{\rho N}(m b)$ & - & $26.7 \pm 2.0$ & $27.7 \pm 1.7$ & $27.9 \pm 2.4$ & $24.5 \pm 1.9$ & $23.5 \pm 2.5$ \\
\hline$\gamma_{p}^{2} / 4 \pi$ & - & $0.57 \pm 0.10$ & $0.59 \pm 0.08$ & $0.58 \pm 0.11$ & $0.50 \pm 0.09$ & $0.50 \pm 0.11$ \\
\hline
\end{tabular}

with Eq. (2), inserting the measured values of $R(A), m_{\rho}, \Gamma_{0}$, and $B G$. Table I summarizes some typical cross sections $\Sigma_{n}=\left(d \sigma_{n} / d t\right)\left(\theta=0^{\circ}\right.$, $\left.\left|t_{\|}\right|=0.002, p=6.54\right)$ with $k_{\max }=7.4 \mathrm{GeV}$; the index $n$ refers to the mass distributions $R_{n}(m)$ used to fit the data. The errors in the cross sections include uncertainties in $R(A), m_{\rho}, \Gamma_{0}$, and $f_{\mathrm{BG}}$. Also listed are typical values of $\chi^{2}(A) /$ $D F(A)$ for all the fits. Both the "Ross-Stodolsky" $R_{1}(m)\left[\right.$ with $\chi^{2}(A) / D F(A) \simeq 1.2$, see Fig. 1] and the "Söding" $R_{2}(m)$ and $R_{3}(m)$ yield decisively better fits to the data and we choose them as our best results. Fig. 3 shows the results for $\Sigma_{1}$.

(5) To determine $\sigma_{\rho N},\left|f_{0}\right|^{2}$, and $\gamma_{\rho}{ }^{2} / 4 \pi$ the cross sections were compared with Eq. (3) inserting $\beta=-0.2$ and our measured values of $R(A)$. The results corresponding to the various BreitWigner forms $R_{n}(m)$ are listed in Table I. These values are seen to be consistent with each other and we choose representative values based on $\Sigma_{1}$ which are

$$
\begin{aligned}
& \sigma_{\rho N}=26.7 \pm 2.0 \mathrm{mb}, \\
& \left|f_{0}\right|^{2}=\left.\frac{d \sigma}{d t}\right|_{t=0} \quad(A=1)=118 \pm 6 \mu \mathrm{b} /(\mathrm{GeV} / c)^{2},
\end{aligned}
$$

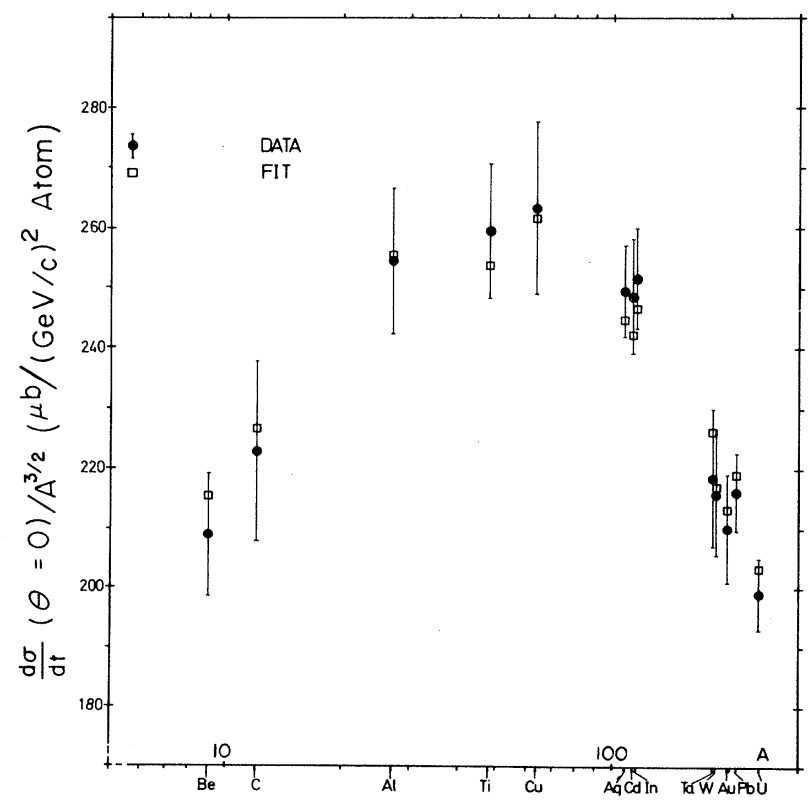

FIG. 3. The values for $\Sigma_{1}$ together with the best fit to these results from which we obtain $\gamma_{\rho}{ }^{2} / 4 \pi=0.57$ \pm 0.10 and $\sigma \rho_{N}=26.7 \pm 2.0 \mathrm{mb}$. 
and

$$
\gamma_{\rho}^{2} / 4 \pi=0.57 \pm 0.10
$$

(see Fig. 3).

We consider the following to be improvements compared with earlier work ${ }^{2,3}$ : (a) This experiment has such good statistics that it enables us to make a more detailed measurement of the background, the $\rho$ line shape, the nuclear physics parameters, etc. than was previously possible.

(b) This experiment used twice as many elements. In particular, there are eight elements with $A$ $>100$ compared with one or two elements of previous experiments. The large amount of heavynuclei data enables us to obtain reliable results with the Margolis model $^{9}$ which applies best to heavy nuclei. (c) The effects of the real part of $\beta$ and nuclear correlations are included in the analysis.

We present the following examples of consis tency checks made to the data (Table I) and analysis:

(1) The directly measured cross sections $d^{2} \sigma /$ $d \Omega d m$ agree with the values obtained by Asbury et al. ${ }^{2}$

(2) To ensure that our results are not sensitive to the nuclear physics of light nuclei, we have analyzed the $A$ dependence by eliminating the data on $\mathrm{Be}, \mathrm{C}$, and $\mathrm{Al}$. We also analyzed the data by systematically eliminating each element in turn. The results did not change.

(3) We have fitted various selected subsets of the data with restricted $m, p, t$ ranges. The results were in good agreement with all values quoted.

(4) We analyzed the data with a large set of Breit-Wigner mass distributions and the results were consistent with each other (five examples are listed in Table I).

(5) A change in $s(=0.545 \mathrm{~F})$ of $\pm 10 \%$ changes the results by $<2 \%$.

(6) A change in $\beta$ of $\pm 50 \%$ changes $\gamma_{\rho}^{2} / 4 \pi$ by $10 \%$ and changes $R(A)$ by $<1 \%$. A change in $R(A)$ by $5 \%$ changes $\sigma_{\rho N}$ by $1.0 \mathrm{mb}$.

(7) The measured radii $R(A)=(1.12 \pm 0.02) A^{1 / 3} \mathrm{~F}$ are in agreement with the commonly used values of $R(A)=1.14 A^{1 / 3} \mathrm{~F}$ (the latter being a $\pm 10 \%$ value). ${ }^{16}$

(8) The value $\left|f_{0}\right|^{2}$ agrees with our measured $H_{2}$ cross section $\left.(d \sigma / d t)\right|_{t=0}=119 \pm 7 \mu \mathrm{b} /(\mathrm{GeV} / c)^{2}$ at $6.0 \mathrm{GeV} / c .{ }^{18}$

(9) The value of $\gamma_{\rho}^{2} / 4 \pi$ is consistent with the independent determination from measurement of total hadronic cross sections $\sigma_{\gamma A}$ from DESY, ${ }^{19}$ from the analysis of other vector meson data from complex nuclei by Margolis, ${ }^{20}$ and with the results of Asbury et al. ${ }^{2,21}$

Conclusions: (I) This experiment provides a measurement of the strong-interaction nuclear density parameters. (II) The measured $\rho$-nucleon cross section agrees with the $\pi$-nucleon cross section in the same energy region $(\simeq 25 \mathrm{mb})$. (III) Comparing our values for $\gamma_{\rho}^{2} / 4 \pi$ with the value determined from $\rho \rightarrow e^{+} e^{-}$decay $^{22}$ of $0.52_{-0.06}^{+0.07}$ we conclude that to an accuracy of $\pm 20 \%$, the $\rho$-photon coupling strength does does not depend on $m_{\gamma}$, the photon mass, in the range 0 $<m_{\gamma}<m_{\rho}$. (IV) The results on $\gamma_{\rho}^{2} / 4 \pi$ and $\sigma_{\rho N}$ are in good agreement with the predictions of vector dominance model. ${ }^{4}$

We are greateful for the support of Professor W. Jentschke, Professor V. F. Weisskopf, Professor P. Demos, Professor A. G. Hill, and Professor H. Joos, who made this collaboration possible. We wish to thank Dr. S. D. Drell, Dr. J. S. Trefil, Dr. B. Margolis, Dr. A. Dar, Dr. R. Wilson, Dr. E. Lohrmann, Dr. M. Nauenberg, Dr. H. Quinn, and Dr. J. Weber for interesting comments. We are greateful to Dr. H. O. Wuester, Dr. D. Lublow, Mr. H. Kumpfert, Miss I. Schultz and Mr. P. Berges for technical assistance.

\footnotetext{
${ }^{1}$ The original work, showing coherent diffractive nature of $\rho$ production, was done by L. J. Lanzerotti et al., Phys. Rev. 166, 1365 (1968).

${ }^{2} \mathrm{~J}_{0}$ G. Asbury et al., Phys. Rev. Letters 19, 865 (1967), and 20, 227 (1968).

${ }^{3}$ G. McClellan et al., Phys. Rev. Letters 22, 377 (1969); J. Swartz and R. Talman, Phys. Rev. Letters $\underline{23}, 1078$ (1969); F. Bulos et al., Phys. Rev. Letters $\underline{22}, 490$ (1969).

${ }^{4} \mathrm{~J}$. J. Sakurai, in Lectures in Theoretical Physics, edited by W. E. Brittin et al. (Gordon and Breach, New York, 1968), Vol. XI; H. Joos, Acta Phys. Austriaca, Suppl. IV, 320 (1967).

${ }^{5}$ J. G. Asbury et al., Phys. Rev. 161, 1344 (1967).

${ }^{6}$ M. J. Longo and B. J. Moyer, Phys. Rev. 125, 701 (1962).

${ }^{7} \mathrm{H}$. Alvensleben et al., DESY Report No. 69/50, December 1969 (unpublished).

${ }^{8}$ In obtaining Fig. 2, the experimental cross section $d \sigma / d \Omega d m$ was divided by $p^{2}\left(f_{c}+f_{\text {in c }}\right)$. We thus remove the $\rho$-production mechanism (but not the background) from the mass spectra.

${ }^{9}$ K. S. Kölbig and B. Margolis, Nucl. Phys。 B6, 85 (1968). Gottfried and Julius have shown that corrections arising from $\rho^{0}$ instability are quite small. See K. Gottfried, Bull. Am. Phys. Soc. 13, 175 (1968).

${ }^{10}$ G. v. Bochmann, B. Margolis, and L. C. Tang, Phys. Letters 30B, 254 (1969). This causes a $4 \%$ correction to our final results.
} 


\footnotetext{
${ }^{11}$ We are grateful to A. Dar, B. Margolis, and R. Wilson for pointing out the importance of this term. For experimental determination of $\beta$, see J. Weber, thesis, DESY, 1969 (unpublished).

${ }^{12} J$. S. Trefil, Nucl. Phys. B11, 330 (1969).

${ }^{13} \mathrm{~J}$. D. Jackson, Nuovo Cimento 34, 1644 (1964) .

${ }^{14}$ M. Ross and L. Stodolsky, Phys. Rev. 149, 1172 (1966); G. Kramer and J.L. Uretsky, Phys. Rev。181, 1918 (1969); P. Söding, Phys. Letters 19, 702 (1966).

${ }^{15}$ F. James and M. Roos, CERN Report No. 67/623/1 (unpublished).

${ }^{16} \mathrm{~J}$. R. Glauber and G. Matthiae, Istituto Superiore de Sanità, Roma, Report No. 67/16 (unpublished); R. Wilson, Lettere Nuovo Cimento 1, 952 (1969).

${ }^{17}$ R. Hofstadter, Ann. Rev. Nucl. Sci. 4, 231 (1957).
}

\author{
${ }^{18} \mathrm{H}$. Alvensleben et al., Phys. Rev. Letters $\underline{23}, 1058$ \\ (1969). \\ ${ }^{19} \mathrm{H}$. Meyer, private communication. Also E。Lohr- \\ mann, DESY Report No. 69/21 (unpublished). \\ ${ }^{20} \mathrm{G}$. Bochmann and B. Margolis, Phys. Rev. Letters \\ 23, 939 (1969). \\ ${ }^{21}$ The original value obtained by Asbury et al. was \\ $0.45 \pm 0.10$. This was obtained using a different nuclear \\ model and did not take into account $\beta$ and $\xi$ (they are \\ opposite effects) which could produce $\mathrm{a} \simeq 15 \%$ effect. \\ ${ }^{22}$ Samuel C. C. Ting, in Proceedings of the Fourteenth \\ International Conference on High Energy Physics, \\ Vienna, Austria, September 1968, edited by J. Prentki \\ and J. Steinberger (CERN Scientific Information Ser- \\ vice, Geneva, Switzerland, 1968).
}

\title{
DETERMINATION OF STRONG-INTERACTION NUCLEAR RADI*
}

\author{
H. Alvensleben, U. Becker, William K. Bertram, M. Chen, K. J. Cohen, T. M. Knasel, \\ R. Marshall, D. J. Quinn, M. Rohde, G. H. Sanders, H. Schubel, and Samuel C. C. Ting
}

Deutsches Elektronen Synchrotron, Hamburg, Germany, and Department of Physics and Laboratory for Nuclear Science, Massachusetts Institute of Technology, Cambridge, Massachusetts 02139 (Received 27 February 1970)

\begin{abstract}
We present results on the determination of a set of strong-interaction nuclear radii using the photoproduction of neutral rho mesons. Analysis of $10^{6}$ events from 13 complex nuclei yields the Woods-Saxon radii $R(A)=(1.12 \pm 0.02) A^{1 / 3} \mathrm{fm}$.
\end{abstract}

In recent years considerable theoretical ${ }^{1}$ and experimental ${ }^{2,3}$ effort has been made using nuclei as a tool to study elementary-particle physics. Attempts have been made to study the properties of elementary particles (stable and unstable) interacting with nuclear matter, such as the rho-nucleon cross section, the vector-dominance model, etc. A knowledge of the nuclear density distribution is essential in order to make a quantitative comparison between theory and experiment and to extract physical quantities from the data. To date, the nuclear density distributions have been assumed to be those determined from electron ${ }^{4}$ or proton ${ }^{5}$ scattering on complex nuclei. When using the electromagnetic nuclear radii, one assumes that the nuclear density distributions seen by strongly interacting particles are the same as those seen by the electron, an assumption which has not been justified. When using proton-scattering data to determine the radii, the problem of interference between the proton and the Coulomb field of the nuclei arises. Because of the importance of obtaining a reliable set of nuclear density distributions for strong interactions, we have performed an experiment specifically designed to measure strong-interaction radii with neutral particles.
This experiment measured the photoproduction of $\rho^{0}$ from nuclei, ${ }^{2}$

$$
\gamma+A \rightarrow A+\rho^{0}-A+\pi^{+}+\pi^{-} .
$$

It was carried out at the 7.5-GeV DESY synchrotron using a bremsstrahlung beam and a magnetic spectrometer with a resolution of $\Delta m= \pm 15$ $\mathrm{MeV} / c^{2}, \Delta p= \pm 150 \mathrm{MeV} / c$, and $\Delta t_{\perp}= \pm 0.001$ $(\mathrm{GeV} / c)^{2}$. We have investigated 13 elements: $\mathrm{Be}, \mathrm{C}, \mathrm{Al}, \mathrm{Ti}, \mathrm{Cu}, \mathrm{Ag}, \mathrm{Cd}$, In, Ta, W, $\mathrm{Au}, \mathrm{Pb}$, and $U$. The measurements cover 20 intervals in the di-pion mass $(m)$ from 400 to $1000 \mathrm{MeV} / c^{2}$, six intervals in the momentum $(p)$ from 4.8 to $7.2 \mathrm{GeV} / c$, and 20 intervals in the transverse momentum transfer to the nucleus $\left(t_{\perp}\right)$ from 0.0 to $-0.04(\mathrm{GeV} / c)^{2}$. These data form a four-dimensional matrix $d^{2} \sigma / d \Omega d m$ with dimensions $\left(A, m, p, t_{\perp}\right)=(13,20,6,20)$ containing approximately $10^{6}$ measured $\pi^{+} \pi^{-}$events. A projection of $2 \%$ of the data (shown in Fig. 1 of the preceding Letter ${ }^{2}$ ) shows that the spectra are dominated by the $\rho$ diffractively produced off the nucleus.

The slopes of the diffraction patterns measure directly the nuclear density distributions. For example, at $t \rightarrow 0$, the diffraction pattern behaves as $e^{a t}$ where $a$ is a measure of the nuclear size. More specifically, we take the nuclear density 